\title{
Impacto del tráfico de equipos durante la cosecha de caña de azúcar (Saccharum officinarum)
}

\author{
Luís A. Rodríguez $^{1} \&$ Jhon J. Valencia ${ }^{1}$
}

\section{RESU MO}

Para determinar el impacto del tráfico sobre el suelo, el cultivo y el consumo energético durante la cosecha de caña de azúcar en el valle del río Cauca (Colombia), se establecieron experimentos de cuatro repeticiones con diferentes sistemas de cosecha. En cada sitio se cosecharon mecánicamente parcelas con vagones de auto volteo, HD8000, HD 12000 y HD20000, se evaluaron por la intensidad de tráfico (IT), el pisoteo directo sobre la cepa, la resistencia a la penetración y el consumo energético. Vagones grandes y pesados causaron mayor IT y mayor efecto por compactación y pisoteo. La IT varió entre 241 y $317 \mathrm{Mg} \mathrm{km} \mathrm{ha-1}$. El pisoteo en la cabecera varió de 8 a $18 \mathrm{~m}$ por surco y sobre la cepa los vagones pisaron entre 5 y $24 \%$ de su ancho. Hubo diferencias no significativas en productividad hasta de $13,9 \%$ favorable a los vagones livianos. En cosecha semimecánica, realizada con trenes de vagones, disminuyó la IT al rango 60-113 $\mathrm{Mg} \mathrm{km} \mathrm{ha}^{-1}$, pero aumentó el pisoteo en las cabeceras hasta $39 \mathrm{~m}$ por surco, hubo diferencias no significativas en productividad hasta del $4 \%$ entre sistemas de vagones livianos y pesados. Además, los vagones livianos con manejo adecuado de la cosecha, llegan a ser favorables por menor consumo de combustible y emisiones.

Palabras-clave: intensidad de tráfico, pisoteo, vagón, consumo de combustible

\section{Impact of traffic equipment during sugarcane (Saccharum officinarum) harvest}

\begin{abstract}
A B ST RACT
This study was carried out to determine the impact of traffic on soil compaction, crop and energy consumption during the sugarcane harvest in the Cauca river valley (Colombia). Four experiments with four replicates were harvested with different systems. Plots were mechanically harvested with self tipping, HD 8000, HD 12000 and HD 20000 trailers and evaluated by traffic intensity (IT), direct stool traffic, penetration resistance and fuel consumption. Heavy trailers caused a greater effect due to a greater IT and direct stool traffic. IT varied between 241 and $317 \mathrm{Mg} \mathrm{km} \mathrm{ha}^{-1}$. Stool traffic at the end of field varied from 8 to $18 \mathrm{~m}$ per furrow, meanwhile stool traffic along the furrow varied from 5 to $24 \%$. There were no significant differences for productivity up to $13.9 \%$ favoring light trailers. Semi-mechanical harvesting was realized with trains of trailers, IT fell down to a $60-113 \mathrm{Mg} \mathrm{km} \mathrm{ha-1}$ range because a larger area is harvested during one pass of the equipment, but stool traffic increased up to $39 \mathrm{~m}$ per furrow for the longer trains, there was a $4 \%$ non significant difference for productivity from light to heavy trailers. Furthermore, light trailers with an adequate management are better options with lower energy consumption and emissions.
\end{abstract}

Key words: traffic intensity, stool traffic, trailer, fuel consumption 


\section{INTRODUCCION}

La compactación considerada como uno de los factores causante de degradación del suelo, se debe a efectos naturales y al tránsito de equipos, que afecta la calidad del suelo y la sostenibilidad porque interfiere el flujo de agua y aire, el desarrollo de las raíces y finalmente disminuye la productividad. En el cultivo de la caña los problemas de compactación se magnifican durante la cosecha por la alta intensidad de tráfico que demanda la labor y por el tránsito directo sobre la cepa (pisoteo). En el Valle del río Cauca (Colombia) se cultivan 210.000 ha de caña de azúcar con productividad promedio de $117 \mathrm{TCH}$ (toneladas de caña ha ${ }^{-1}$ ) cosechadas con equipos pesados en forma continua durante todo el año, inclusive en épocas húmedas cuando es mas severo el impacto del tráfico de equipos. La principal causa de compactación en suelos agrícolas es el tráfico de máquinas y equipos durante las labores de cultivo y las operaciones de cosecha en especial cuando se realizan en suelos húmedos y con altas presiones sobre el área de contacto suelo-llanta (Botta et al., 2007).

La cuantificación de los efectos del tráfico usualmente incluye peso de vehículos e implementos, distancia recorrida, presión de contacto, área cubierta por llantas u orugas y número de pases (Kuipers \& Zande, 1994). En la cosecha de caña son importantes las cargas que se desplazan y las presiones de contacto, ambos factores definen la compactación en el perfil del suelo y son las influencias dominantes en términos del daño potencial porque determinan el nivel inicial de esfuerzos en la superficie, mientras que la carga por llanta define la tasa de disminución de los esfuerzos con respecto a la profundidad (Chamen et al., 2003). La condición final del suelo depende de los esfuerzos transmitidos por los equipos durante la cosecha. El tráfico de máquinas agrícolas modifica la condición del suelo, aumenta la densidad, disminuye el diámetro medio ponderado de los agregados y la macro porosidad en el entresurco con relación a la línea de plantío y esto, causa degradación acumulativa de la calidad del suelo a través del tiempo (Botta et al., 2004).

La intensidad de tráfico (IT) es un parámetro adecuado para definir la distribución del tráfico sobre el campo; en la actualidad se puede utilizar el sistema de información geográfica para su determinación precisa, es útil para comparar sistemas de mecanización o cosecha y sus posibles efectos en la productividad. Botta et al. (2007) concluyeron que las diferencias significativas encontradas en la producción de maíz se debieron al daño que la alta intensidad de tráfico causó sobre la estructura del suelo en la superficie, IT de 180, 120 y 60 Mg km ha ${ }^{-1}$ causaron caídas en producción de 38, 22,6 y 9,8\% respectivamente.

Diferentes alternativas han sido planteadas para disminuir los efectos del tránsito de equipos: tráfico controlado, disminución del número de pases y de la intensidad de tráfico o minimizar la presión de contacto disminuyendo la carga por eje o usando llantas de gran superficie de contacto (Hamza \& Anderson, 2005). Nagaoka et al. (2003) evaluaron llantas diagonales y radiales y concluyeron que el tipo de llanta también tiene influencia significativa en la compactación del suelo.
Además de aspectos relacionados al tránsito de equipos, el suelo aporta factores de gran importancia por su susceptibilidad a la compactación y su capacidad para soportar esfuerzos según la textura, el contenido inicial de humedad y el esfuerzo de precompresión, Saffih et al. (2009) relacionaron matemáticamente estos factores y mostraron que a mayor contenido de arcilla mayor es la capacidad del suelo para soportar esfuerzos. En suelos arcillosos y limosos el contenido de agua es el factor más importante mientras que en suelos arenosos los parámetros mecánicos son menos dependientes de la condición inicial de humedad y mas dependientes de la densidad aparente inicial. El cultivo y el diseño de campo también aportan elementos que se interrelacionan con los equipos y generan factores medibles como el pisoteo directo sobre la cepa que depende de las dimensiones de los equipos, el entresurco, el ancho de los callejones y el ancho de la cepa.

Este trabajo se ejecutó con el objetivo de plantear alternativas que permitan aminorar los efectos del tráfico, en cosechas comerciales se cuantificaron los factores que generan compactación y pisoteo, los resultados permitieron plantear alternativas que incluyen factores relacionados a los equipos y el campo: reducir los esfuerzos transmitidos al suelo a través de vagones de menor peso y capacidad, usar llantas mas adecuadas, relacionar el diseño de los vagones y el diseño de campo, limitar los trenes de vagones y supervisar las labores de cosecha. Estas alternativas contribuyen a disminuir la compactación y el pisoteo y favorecen el medio ambiente disminuyendo el consumo energético y las emisiones de gases de efecto invernadero.

\section{MATERIAL Y METODOS}

Este trabajo fue desarrollado por el Centro de Investigación de la caña de azúcar de Colombia, cuya estación experimental se localiza a $3^{\circ} 30^{\prime} 54^{\prime \prime}$ latitud norte, $76^{\circ} 33^{\prime} 64^{\prime \prime}$ longitud Oeste y $1025 \mathrm{~m}$ de altitud sobre el nivel del mar. Para identificar el efecto del tránsito de equipos de cosecha de caña en el rendimiento del cultivo se deben medir factores relacionados al suelo y al cultivo como la compactación inducida y el pisoteo que dependen del tamaño y capacidad de los equipos, el diseño de campo y el sistema de cosecha. Aunque la mayor causa de compactación se relaciona al peso de los equipos, es útil tener información sobre la distribución del tránsito en el campo durante ciertas labores o en el ciclo del cultivo (Kroulik et al., 2009). Esa distribución es mas importante en las labores que manejan altas cargas como en la cosecha de caña, conocerla implica el seguimiento al tránsito de los equipos, y se cuantifica a través de la intensidad de tráfico (IT) que involucra el efecto de cargas, distancias recorridas y área de la superficie afectada o beneficiada, que se expresa en $\left(\mathrm{Mg} \mathrm{km} \mathrm{ha}^{-1}\right)$; para su evaluación, se establecieron cuatro experimentos en lotes comerciales en los Ingenios Riopaila-Castilla, Manuelita e Incauca. En cada sitio se cosecharon parcelas con diferentes sistemas de cosecha definidos por el tipo de vagón (tratamiento), se utilizaron los equipos listados en la Tabla 1. 
Tabla 1. Equipos utilizados para evaluaciones de tráfico en cosechas comerciales

\begin{tabular}{llrr}
\hline \multirow{2}{*}{ Equipo } & \multirow{2}{*}{ Rodamiento } & Tara & Peso bruto \\
\cline { 3 - 4 } & & \multicolumn{2}{c}{ (kN) } \\
Cosechadora & Orugas & & 180 \\
Alzadora & Llanta 32,1-26 & 90 \\
Tractor 1 (9330) & Llanta 710/70R38 & 160 \\
Tractor 2 (245) & Llanta 710/70R38 & 124 \\
Tractor 3 (8100) & Llanta 710/70R38 & & 90 \\
Vagón autovolteo & Llanta 600/50-22,5 & 60 & 155 \\
Vagón HD12000 & LLanta 23,1-26 & 78 & 260 \\
Vagón HD20000 & LLanta 560/80D26 & 110 & 320 \\
Vagón HD8000 (Caucaseco) & Llanta 23,1-26 & 54 & 154 \\
\hline
\end{tabular}

Se registraron datos y se realizó muestreo bajo el siguiente procedimiento:

Antes de la cosecha:

- Muestreo previo del lote de ensayo (resistencia a la penetración, densidad aparente, contenido de agua, perfil de entresurco).

Durante la cosecha:

- Georeferenciación del movimiento de cada vagón o tren de vagones (entrada al lote, inicio de llenado, fin de llenado, salida del lote).

- Medición de longitudes de surco pisadas por cada vagón o tren de vagones a la entrada y salida del lote.

- Muestreo de ancho de cepa pisada por los equipos a lo largo del surco.

- Selección de puntos de muestreo para medición pos cosecha de resistencia a la penetración y densidad aparente (vagón lleno).

Después de la cosecha:

- Medición de resistencia a la penetración y muestreo para densidad aparente en puntos seleccionados.

- Registro de datos de cosecha.

Durante la cosecha se georeferenciaron cuatro puntos del recorrido de cada vagón: entrada al lote, inicio de llenado, fin de llenado y salida del lote. Con estos puntos se calcularon tres distancias correspondientes a tres estados del vagón dentro del lote, vagón vacío, vagón llenando y vagón cargado. Cada distancia recorrida por el vagón tiene asociada una carga que varía desde su peso propio hasta el peso bruto (vagón + caña). Considerando cargas, distancias y áreas correspondientes se determinó la intensidad de tráfico para cada condición de carga del vagón. Para la intensidad del tráfico total causada por cada sistema de cosecha sobre un área determinada se consideró el efecto de vagón, tractor y máquina (cosechadora o alzadora).

\section{RESULTADOS Y DISCUSION}

Los datos registrados que se utilizaron para determinar y analizar los factores que cuantifican los efectos del tráfico sobre el suelo y el cultivo y sus relaciones fueron: intensidad de tráfico, densidad aparente, resistencia a la penetración, pisoteo, desarrollo del cultivo y productividad en los cuatro sitios experimentales cuyas características se muestran en la Tabla 2. Se realizaron cosechas en época seca (sitios 1 y 2) y cosechas en época moderadamente húmeda (sitios 3 y 4), en estos últimos sitios se cosechó con humedad en el límite plástico (LP) o superior, es decir con suelo en condición plástica que se deforma y comprime fácilmente bajo la acción de la carga de los equipos. También se observa que en todos los sitios la humedad al momento de la cosecha fue superior al contenido óptimo de humedad para máxima compactación, es decir no hubo cosecha en el punto de máxima compactibilidad del suelo.

Los recorridos georeferenciados se pueden graficar como muestra la Figura 1, para nueve vagones, cada línea es el recorrido de un vagón entre la entrada y salida del lote. Las líneas horizontales inferiores son el recorrido del vagón vacío, las líneas horizontales superiores son recorridos del vagón lleno en la distancia de salida, las líneas inclinadas representan la fase de llenado, su pendiente es un indicador de la tasa de llenado con respecto a la distancia recorrida y de la productividad del lote.

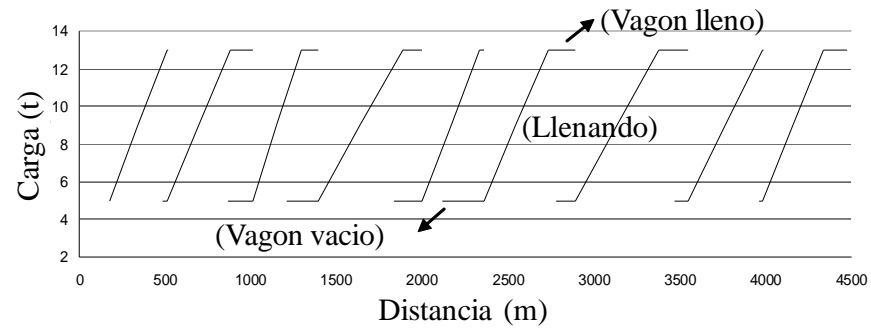

Figura 1. Recorridos de entrada, efectivo y de salida de un conjunto de vagones de autovolteo obtenidos al registrar con G PS el movimiento dentro del campo

Tabla 2. Características de los sitios de cosecha

\begin{tabular}{|c|c|c|c|c|}
\hline Factor & Sitio 1 & Sitio 2 & Sitio 3 & Sitio 4 \\
\hline Tipo de cosecha & Mecánica & Mecánica & Semimecánica & Semimecánica \\
\hline Ingenio & Riopaila-Castilla & Manuelita & Manuelita & Incauca \\
\hline Tipo de suelo & Fluvaquentic Haplustoll & Typic Hapluster & Typic Hapluster & Aquic Haplustoll \\
\hline Familia textural & Francosa-fina & Fina & Fina & Francosa-fina \\
\hline Límite plástico (\%) & 25 & 25 & 23 & 33 \\
\hline Índice de plasticidad (\%) & 9 & 15 & 22 & 16 \\
\hline Contenido de agua promedio (\%) & 20 & 29 & 21 & 32 \\
\hline Densidad máx. $\left(\mathrm{Mg} \mathrm{m}^{-3}\right)$ & 1,67 & 1,52 & 1,59 & 1,34 \\
\hline Humedad óptima (\%) & 17 & 23 & 18 & 20 \\
\hline Entresurco (m) & 1,65 & 1,75 & 1,75 & 1,65 \\
\hline Ancho de cepa (mm) & 500 & 490 & 480 & 490 \\
\hline Longitud de surco (m) & 250 & 180 & 100 & 130 \\
\hline Variedad & CC $85-92$ & CC $85-92$ & CC 93-3895 & CC $84-75$ \\
\hline
\end{tabular}


El registro del tráfico de los vagones también permite determinar la distribución del recorrido de los equipos, para los vagones de la Figura 1 en una cosecha con surcos de $250 \mathrm{~m}$ de longitud, se tienen los siguientes promedios: distancia de entrada $140 \mathrm{~m}$, distancia de cosecha $388 \mathrm{~m}$, distancia de salida $87 \mathrm{~m}$ y distancia promedio transitada por un vagón dentro del campo $615 \mathrm{~m}$, esto significa que en promedio el $23 \%$ del recorrido dentro del campo fue en vacío a la entrada, el $63 \%$ recorrido efectivo cosechando y el $14 \%$ fue recorrido de salida, en total el $37 \%$ son recorridos adicionales o muertos.

La condición ideal se tiene cuando estos recorridos son mínimos y se obtiene al correlacionar la capacidad del vagón y la productividad con longitud del surco a través del diseño de campo, también se pueden minimizar a través del manejo de la cosecha respecto al llenado del vagón. Los recorridos muertos representan incremento en consumos de combustible, emisiones y tiempo extra en el trabajo de las máquinas.

En cosecha mecánica las intensidades de tráfico son muy altas porque el área cosechada es pequeña, se cosecha un surco en cada dirección de viaje, un alto porcentaje de los entresurcos es pisado dos veces por los equipos. La Figura 2, muestra las IT durante una cosecha mecánica con tres sistemas diferentes (vagones HD20000, HD12000 y autovolteo).

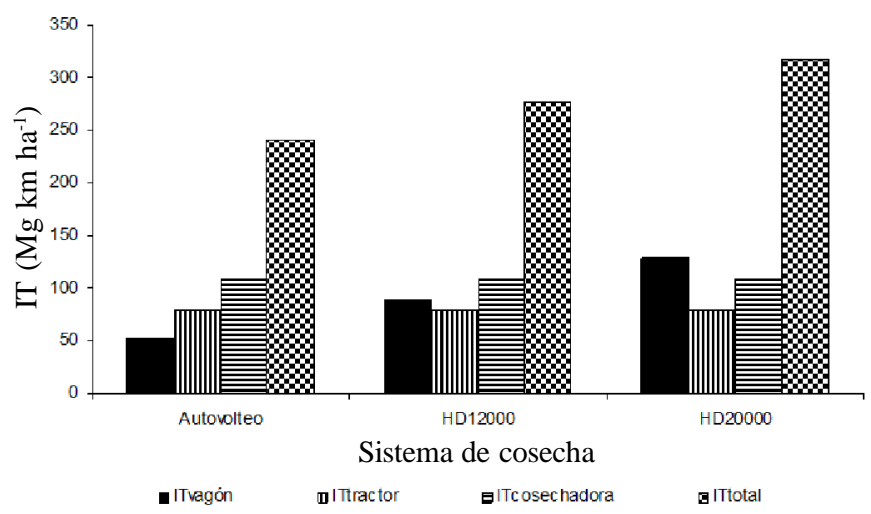

Figura 2. Intensidades de tráfico (IT) causadas por diferentes sistemas de cosecha con vagones HD20000, HD 12000 y autovolteo

La IT total varió entre $317 \mathrm{Mg} \mathrm{km} \mathrm{ha}^{-1}$ para la cosecha con vagón $\mathrm{HD} 20000$ a $241 \mathrm{Mg} \mathrm{km} \mathrm{ha}^{-1}$ para el sistema con vagón de autovolteo. La diferencia se debe al tipo de vagón, a los recorridos muertos y al tamaño del tractor en cada sistema de cosecha. Estos valores son un indicador del efecto potencial del tráfico de los equipos sobre el suelo y el cultivo.

En cosecha semimecánica (corte manual, alce mecánico) las intensidades de tráfico son menores porque el área cosechada es mayor, se alzan seis surcos en cada desplazamiento de los equipos. En épocas secas o normales se cosecha con trenes hasta de cinco vagones (HD8000, HD12000, HD20000), los recorridos muertos se incrementan dependiendo de la longitud de cada tren. La Figura 3, muestra la IT para trenes de 3 y 5 vagones HD12000, HD20000 y HD8000, se observa la influencia del número de vagones y el peso sobre la IT que incrementó de 60 a $80 \mathrm{Mg} \mathrm{km} \mathrm{ha}^{-1}$ al pasar de 3 a 5 vagones en el tren HD8000. El mayor valor registrado por los vagones HD20000 se debe especialmente a la mayor carga, que registran pesos brutos entre 270 y $290 \mathrm{kN}$, lo cual representa cargas entre 90 y 100 $\mathrm{kN} \mathrm{eje}{ }^{-1}$, que son muy superiores a los límites de carga preventivos de compactación severa en las capas sub superficiales del suelo, $60 \mathrm{kN} \mathrm{eje} \mathrm{e}^{-1}$ (Hakansson et al., 1988).

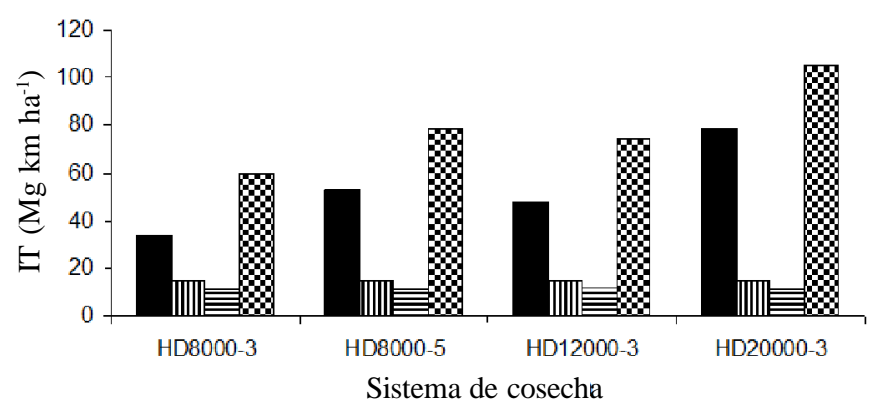

- IT vagon $\quad$ IT tractor EIT alzadora $\mathbf{8}$ total Figura 3. Intensidad de tráfico causada por sistemas de cosecha con trenes de 3 y 5 vagones HD 8000, HD 12000 y HD20000

Trenes de tres vagones HD12000 causan menor IT que los trenes de HD20000 pero presentan la misma situación respecto a las cargas, con pesos brutos hasta de $250 \mathrm{kN}$ que transmiten al piso entre 80 y $125 \mathrm{kN} \mathrm{eje}^{-1}$ debido a que tienen solo dos ejes. Los vagones HD8000 son más conservativos en la transmisión de cargas al suelo llegando hasta $80 \mathrm{kN} \mathrm{eje}^{-1}$, por lo cual aminoran la compactación en el subsuelo.

Los daños en la cosecha no son solo por compactación, también se deben al pisoteo directo sobre el cultivo, es importante la magnitud del área pisada, Kroulik et al. (2009) mostraron que para cualquier cultivo con laboreo mecanizado, el 95,3\% del área es pisada por lo menos una vez durante cada ciclo. En la cosecha mecánica de caña, cosechadora, vagones y tractores pisan en cada pase un área equivalente al 120 $138 \%$ del área cosechada para entresurcos entre 1,5 y $1,75 \mathrm{~m}$ y además entre 45 y $65 \%$ de los entresurcos reciben doble pase de los equipos dependiendo del ancho de la melga cosechada. En cosecha semimecánica los equipos pisan entre el 26 y $30 \%$ del área cosechada. Al transitar sobre el surco se incrementan la densidad aparente, el grado de compacidad y la resistencia al cono y además se reduce la conductividad saturada en la zona del surco en comparación con el entresurco (Braunack et al., 2006a).

El tránsito directo sobre la cepa se presenta en las cabeceras de los lotes y a lo largo del surco. El primero depende principalmente del ancho de los callejones y la longitud de los equipos, también influyen la trocha, el entresurco y el ancho de la cepa. En cosecha mecánica las longitudes de surco pisadas en las cabeceras llegaron a $14,8 \mathrm{~m}$ por surco en lotes con callejones de $8 \mathrm{~m}$ en ambos extremos como muestra la Figura 4, con callejones de $12 \mathrm{~m}$ en un extremo dicha longitud bajó 12,9 m, con el mismo vagón. En cosecha semimecánica realizada generalmente con trenes de vagones se incrementan las longitudes de surco pisado en las cabeceras. Cosechando con trenes de 5 vagones HD8000 y 3 vagones HD12000 o HD20000, las cabeceras pisadas alcanzaron 39 y $32 \mathrm{~m}$ respectivamente en lotes de callejones de $6 \mathrm{~m}$ en ambos extremos. Al disminuir a trenes de 2 vagones convencionales, 


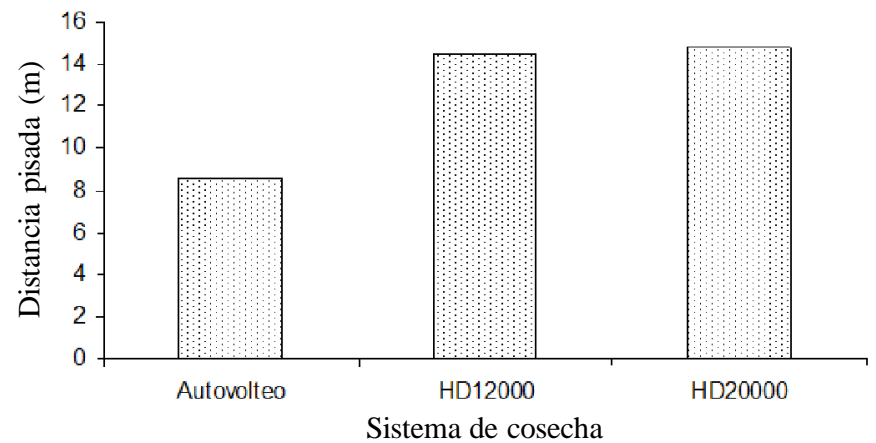

Figura 4. Pisoteo por surco en las cabeceras, cosecha mecánica, ancho de callejones $8 \mathrm{~m}$, entresurco 1,65 m, ancho de cepa $0,5 \mathrm{~m}$

el pisoteo en cabeceras disminuyó a 10 m por surco en lotes con callejones de $8 \mathrm{~m}$.

El pisoteo directo sobre la cepa a lo largo del surco se debe fundamentalmente a las diferencias entre la trocha de los equipos y el entresurco, también influyen el ancho de la cepa y la sección de las llantas. El efecto del pisoteo sobre la cepa es mas crítico en la cosecha mecánica porque un alto porcentaje de los entresurcos es pisado dos veces por la cosechadora; el tractor y el vagón, la diferencia entre los parámetros del cultivo (distancia entre surcos) y de los equipos (trocha) es una causa potencial para que ocurra compactación sobre el surco con posibles efectos adversos sobre la producción (Braunack \& McGarry, 2006b). Los vagones convencionales con trochas de 2,0 a 2,15 m, llantas de 587 o 560 mm de sección pisan hasta el $5 \%$ del ancho de la cepa en cultivos a 1,75 m entre surcos y alcanzan hasta el $24 \%$ en cultivos a $1,65 \mathrm{~m}$.

Este es uno de los factores mas utilizados para referenciar y detectar eventos de compactación. Iaia et al. (2006) encontraron que en caña de azúcar la RP depende del tipo de suelo y el número de corte, mientras que Terminiello et al. (2000) encontraron que la compactación inducida por el tráfico de vehículos se detecta con mayor claridad utilizando la RP en lugar de la densidad aparente.

Las RP es altamente influenciada por el contenido de agua, en suelos arcillosos secos la cohesión entre partículas y la resistencia a la penetración son altas, como se observa en las mediciones realizadas en la cosecha semimecánica con trenes de cinco vagones HD8000 y tres vagones HD12000 o HD20000 mostradas en la Figura 5, en suelo Typic Haplusters de textura arcillosa y $21 \%$ de contenido de agua promedio en el lote de ensayo durante la cosecha. Antes de la cosecha y a $0,20 \mathrm{~m}$ de profundidad la RP era superior a 2,0 MPa y llegaba a 3,3 MPa a $80 \mathrm{~cm}$, estos valores son indicadores de la compactación residual por efecto de pases de máquinas durante el ciclo del cultivo y compactación natural debido especialmente a fenómenos de expansión y contracción de la masa de suelo por cambios en la humedad. Los niveles de compactación se incrementan durante la cosecha por dos causas fundamentales, el peso de los equipos y su pase repetido. Los mayores incrementos en RP se presentan en el subsuelo para los tres sistemas de cosecha a causa de las altas cargas, vagones HD12000 y HD20000 cargados con caña larga transportan entre 100 y $115 \mathrm{kN} \mathrm{eje}^{-1}$, estas cargas son superiores a los límites definidos como preventivos de compactación en el subsuelo. La resistencia a la penetración llega a valores altos de 5,3 MPa, y se observa mayor tendencia al incremento a partir de $0,60 \mathrm{~m}$ para los tres sistemas de cosecha como consecuencia de la compactación acumulada en el subsuelo. El mayor registro en la RP causado por el tránsito del tren de cinco vagones HD8000 muestra el efecto del pase repetido de cargas en la compactación inducida.

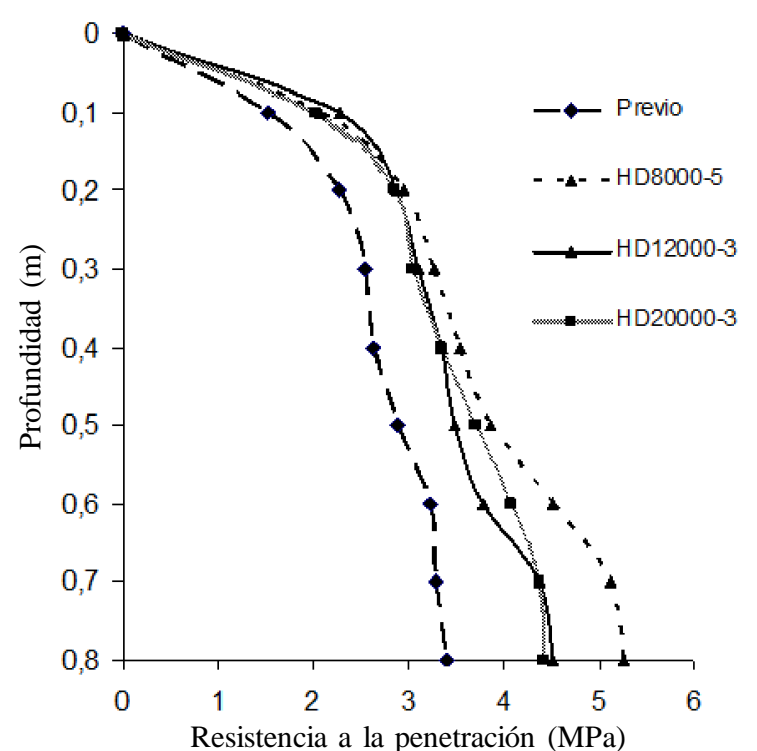

Figura 5. Resistencia a la penetración en cosecha semimecánica con trenes de vagones

La cosecha mecánica se realizó en lotes con mayor contenido de agua lo que disminuyó la resistencia a la penetración por el efecto lubricante del agua. La tendencia a expandir los agregados en suelos arcillosos y el incremento en la plasticidad a medida que aumenta el contenido de agua del suelo, es una condición que se muestra en la Figura 6, en suelo arcilloso de LP $25 \%$, IP $15 \%$ y contenido de agua promedio de $29,3 \%$, el suelo hace uso de sus características plásticas y es fácilmente deformable tanto a la acción de las cargas de los equipos de cosecha como a la acción del cono del penetrómetro. Se observa la tendencia a mantener valores de RP casi constantes a partir de 0,3 m de profundidad, el vagón HD12000 causó mayor efecto sobre el suelo porque con solo dos ejes transmite al suelo mayores cargas.

Los efectos del tráfico de equipos durante la cosecha se pueden cuantificar a través de la productividad del ciclo siguiente, factor en el que se reflejan los efectos de la compactación y el pisoteo. La Tabla 3 muestra los factores influyentes y resultados en dos sitios de cosecha mecánica con tres y cuatro tratamientos y cuatro repeticiones por sitio. En el primero se evaluaron tres sistemas de cosecha compuestos de cosechadora, tractor y vagón (HD20000, HD12000 o autovolteo).

Se registraron diferencias estadísticamente significativas en los tres factores determinantes del efecto de la cosecha sobre el suelo y el cultivo: intensidad de tráfico, pisoteo sobre las cabeceras y pisoteo sobre la cepa, vagones de mayor peso propio, capacidad y longitud causan mayor daño, el cual 


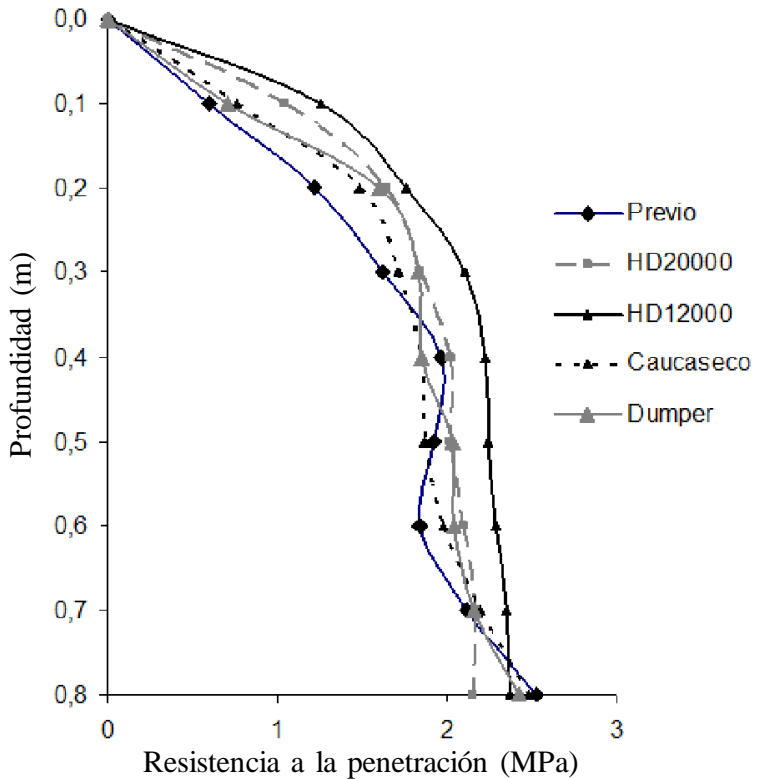

Figura 6. Resistencia a la penetración en cosecha mecánica

finalmente incide en la productividad. En el sitio 1, sistemas de cosecha mecánica con vagones de gran tamaño generaron mayores efectos con $142 \mathrm{TCH}$ mientras que las parcelas cosechadas con vagones pequeños (autovolteo) alcanzaron $164 \mathrm{TCH}$, es una diferencia estadísticamente no significativa del $13,4 \%$ en la que favoreció el uso de sistemas de cosecha con vagones de menor peso que causan menor IT y menor pisoteo.

En el sitio 2 se cosechó a un contenido de agua por encima del límite plástico donde el suelo tiene mayor capacidad de deformación y menor capacidad portante. La distancia entre surcos 1,75 m fue favorable desde en el punto de vista de la cosecha con los vagones grandes que con trochas similares se acomodaron mejor en el entresurco y redujeron notablemente el efecto sobre el cultivo.

El sistema dumper se destaca por alto pisoteo, con una trocha de 2,47 m transita continuamente sobre la cepa pisando hasta el 66\% de su ancho. El mayor efecto lo causó el sistema de cosecha con vagón HD20000 por su alta IT $310 \mathrm{Mg} \mathrm{km} \mathrm{ha}^{-1}$, parcelas cosechadas con ambos sistemas alcanzaron menores producciones con $118 \mathrm{TCH}$.

La cosecha con vagones mas pequeños y de menor peso generaron menores efectos como el vagón HD8000 cuyas parcelas alcanzaron producciones de $137 \mathrm{TCH}$. El vagón HD12000 con IT intermedia y menos pisoteo alcanzó producciones de $127 \mathrm{TCH}$. Desde el sistema de cosecha con vagones livianos y pequeños a la cosecha con vagones grandes y pesados se registraron diferencias estadísticas no significativas en productividad equivalentes al 13,9\%. Los resultados obtenidos en los dos sitios de cosecha mecánica son consistentes en los efectos del tráfico sobre la productividad.

En cosecha semimecánica los efectos del tráfico de equipos son menores, disminuyen la carga por vagón y la IT, sin embargo se realiza con trenes de vagones que incrementan los esfuerzos por su pase repetido y también aumenta el pisoteo en las cabeceras.

La Tabla 4 muestra los resultados en dos sitios (3 y 4 ) de cosecha semimecánica, con tres y cuatro tratamientos y cuatro repeticiones. En el sitio 3 se cosechó con humedad inferior al límite plástico donde el suelo conserva buena capacidad de

Tabla 3. Factores de tráfico y productividad después de cosecha mecánica con sistemas diferentes

\begin{tabular}{|c|c|c|c|c|c|c|}
\hline $\begin{array}{c}\text { Sistema } \\
\text { de cosecha }\end{array}$ & $\begin{array}{l}\text { Rango de } \\
\text { carga (kN) }\end{array}$ & $\begin{array}{c}\text { Recorrido } \\
\text { muerto } \mathbf{m}(\%)\end{array}$ & $\begin{array}{c}\text { IT } \\
\left(\mathrm{Mg} \mathrm{km} \mathrm{ha}^{-1}\right)\end{array}$ & $\begin{array}{c}\text { Pisoteo } \\
\text { cabeceras (m) }\end{array}$ & $\begin{array}{l}\text { Pisoteo } \\
\text { Cepa (\%) }\end{array}$ & $\begin{array}{l}\text { TCH } \\
\left(\mathrm{t} \mathrm{ha}^{-1}\right)\end{array}$ \\
\hline $\begin{array}{l}\text { HD12000 } \\
\text { HD20000 } \\
\text { Autovolteo }\end{array}$ & $\begin{array}{r}78-263 \\
109-320 \\
55-155\end{array}$ & $\begin{array}{r}91(10) \\
164(12) \\
214(37)\end{array}$ & $\begin{array}{l}\text { Sitio } 1 \\
276 b \\
317 c \\
241 a\end{array}$ & $\begin{array}{r}14,6 \mathrm{~b} \\
13,9 \mathrm{~b} \\
8,9 \mathrm{a} \\
\end{array}$ & $\begin{array}{r}10 b \\
24 c \\
0 a \\
\end{array}$ & $\begin{array}{l}142 \mathrm{a} \\
142 \mathrm{a} \\
164 \mathrm{a}\end{array}$ \\
\hline $\begin{array}{l}\text { HD12000 } \\
\text { HD20000 } \\
\text { HD8000 } \\
\text { Dumper }\end{array}$ & $\begin{array}{r}78-263 \\
109-320 \\
55-157 \\
195-275\end{array}$ & $\begin{array}{l}102(10) \\
35(3) \\
88(13) \\
99(20)\end{array}$ & $\begin{array}{r}\text { Sitio } 2 \\
248 a \\
311 b \\
238 a \\
237 a\end{array}$ & $\begin{array}{r}9,0 \mathrm{a} \\
6,4 \mathrm{a} \\
21,7 \mathrm{~b} \\
13,4 \mathrm{~b}\end{array}$ & $\begin{array}{r}0,5 \mathrm{a} \\
2,6 \mathrm{a} \\
15,2 \mathrm{~b} \\
65,8 \mathrm{c}\end{array}$ & $\begin{array}{l}127 a \\
118 a \\
137 a \\
118 a\end{array}$ \\
\hline
\end{tabular}

Medias seguidas de la misma letra no difieren significativamente entre si a un nivel del 0,05, IT - intensidades de tráfico, TCH - toneladas de canã ha-1

Tabla 4. Factores de tráfico y productividad después de cosecha semimecánica con sistemas diferentes de 5,3 y 2 vagones por tren

\begin{tabular}{|c|c|c|c|c|c|c|}
\hline Cosecha con trenes & $\begin{array}{l}\text { Rango de } \\
\text { carga (kN) }\end{array}$ & $\begin{array}{l}\text { Recorrido } \\
\text { muerto (\%) }\end{array}$ & $\begin{array}{c}\text { IT } \\
\left(\mathrm{Mg} \mathrm{km} \mathrm{ha}^{-1}\right)\end{array}$ & $\begin{array}{c}\text { Pisoteo } \\
\text { cabeceras }(\mathrm{m})\end{array}$ & $\begin{array}{l}\text { Pisoteo } \\
\text { Cepa (\%) }\end{array}$ & $\begin{array}{l}\mathrm{TCH} \\
\left(\mathrm{t} \mathrm{ha}^{-1}\right)\end{array}$ \\
\hline $\begin{array}{l}\text { HD12000 (3) } \\
\text { HD20000 (3) } \\
\text { HD8000 (5) }\end{array}$ & $\begin{array}{l}75-247 \\
95-290 \\
55-130\end{array}$ & $\begin{array}{l}73 \\
69 \\
81\end{array}$ & $\begin{array}{c}\text { Sitio } 3 \\
74 a \\
99 \mathrm{~b} \\
79 \mathrm{a}\end{array}$ & $\begin{array}{l}20,5 \mathrm{a} \\
19,0 \mathrm{a} \\
24,5 \mathrm{~b}\end{array}$ & $\begin{array}{r}0,2 \mathrm{a} \\
1,6 \mathrm{~b} \\
14,5 \mathrm{c}\end{array}$ & $\begin{array}{l}140 a \\
134 a \\
138 a\end{array}$ \\
\hline $\begin{array}{l}\text { HD12000 (2) } \\
\text { HD20000 (2) } \\
\text { Autovolteo (2) } \\
\text { Manual }\end{array}$ & $\begin{array}{l}80-223 \\
92-295 \\
80-157\end{array}$ & $\begin{array}{l}70 \\
50 \\
69\end{array}$ & $\begin{array}{c}\text { Sitio } 4 \\
74 a \\
60 a \\
67 a\end{array}$ & $\begin{array}{r}10,5 \mathrm{a} \\
9,8 \mathrm{a} \\
9,2 \mathrm{a}\end{array}$ & $\begin{array}{r}9,7 \mathrm{a} \\
23,7 \mathrm{c} \\
18,2 \mathrm{~b}\end{array}$ & $\begin{array}{l}128 a \\
126 a \\
130 a \\
133 a\end{array}$ \\
\hline
\end{tabular}

Medias seguidas de la misma letra no difieren significativamente entre si a un nivel del 0,05, IT - intensidades de tráfico, TCH - toneladas de canã ha-1 
soporte y los problemas de deformación superficial se minimizan.

Todos los factores relacionados al tráfico de los equipos mostraron diferencias estadísticamente significativas y los mayores efectos se debieron al pisoteo de los vagones en las cabeceras, que se cosechó con trenes de cinco vagones HD8000 y de tres vagones HD12000 o HD20000 que presentaron dificultades de maniobra en los extremos del lote. Además, el pase repetido de vagones incrementa el esfuerzo transmitido al suelo, el mayor efecto se causa al paso del primer vagón, los vagones restantes incrementan el esfuerzo causado tanto a nivel superficial como en el subsuelo, estudios realizados por Cenicaña mostraron que trenes de vagones HD12000 cargados con caña larga incrementan los esfuerzos superficiales a razón de $4,3 \%$ por cada vagón, el efecto del número de pases llega a ser mas importante que el peso en la responsabilidad principal de inducir compactación en el subsuelo (Jorajuria \& Draghi, 2000).

$\mathrm{Al}$ aumentar el número de pases también se incrementa la IT y aumentan la compactación y la profundidad de la capa compactada en forma independiente de la carga (Balbuena et al., 2000). El pisoteo sobre la cepa fue bajo para los trenes con vagones grandes porque sus trochas se ajustaron al cultivo (1,75 m de entresurco), no ocurrió igual con el vagón HD8000 que alcanza a pisar el $15 \%$ de la cepa, por diferencias entre la trochas delantera y trasera. El pisoteo en las cabeceras con trenes de vagones se incrementó en promedio hasta $24,5 \mathrm{~m}$ por surco por las dificultades de maniobra en las salidas y entradas al lote.

Esta es una causa importante para las diferencias en producción entre los tres sistemas de cosecha que variaron desde 140 TCH para los trenes de vagones HD12000 hasta 134 TCH para los trenes de vagones pesados (HD20000), esto representa una diferencia no significativa del $4 \%$ entre los tres sistemas de cosecha.

En el sitio 4 cosecha semimecánica se realizó con humedad correspondiente al límite plástico. Los resultados muestran al tren de vagones mas pesado con la menor IT, porque coincidieron el diseño de campo (longitud del surco) con la capacidad del tren de vagones, los recorridos muertos fueron nulos para el tren de vagones no así para los vagones individuales, al disminuir la IT causada por vagón y tractor, disminuyen también los efectos sobre el suelo y el cultivo. Un factor de gran efecto es el pisoteo sobre la cepa que alcanza el $24 \%$ para los vagones grandes y el $18 \%$ para el vagón de autovolteo que para este caso es una versión para caña larga de $80 \mathrm{kN}$ de peso y $2,85 \mathrm{~m}$ de trocha. El pisoteo en las cabeceras presenta valores muy similares y cercanos a $10 \mathrm{~m}$ por surco, estos son valores normales en lotes con callejones de $8 \mathrm{~m}$ en ambos extremos. No se presentó diferencia estadísticamente significativa en producción con valores muy similares entre 126 y $130 \mathrm{TCH}$ para los tres sistemas de cosecha. La mayor productividad, $133 \mathrm{TCH}$ se obtuvo en parcelas donde la caña fue retirada manualmente del campo, con respecto a este testigo la caída en producción por efecto del tránsito alcanzó 5,3\% para los vagones pesados.

Estos resultados muestran que las características de los vagones crean diferencias en los efectos que cada sistema de cosecha causa sobre el suelo y el cultivo, vagones de mayor tamaño crean mayor intensidad de tráfico y son potencialmente mas propensos a pisar sobre la cepa e inducir mayores efectos sobre el suelo y el cultivo. Dichos efectos se manifiestan con menor severidad en la cosecha semimecánica (Meyer, 2001) debido inicialmente a que se inducen menores intensidades de tráfico. Este factor además puede disminuir con manejo adecuado de la cosecha, a través del cual se pueden eliminar los recorridos muertos a la entrada y salida del lote.

Como cualquier labor agrícola, el movimiento de los vagones de cosecha demanda un consumo energético y genera emisiones, ambos factores pueden disminuir a través del manejo adecuado de la cosecha y la correlación entre equipos y diseño de campo para que el ciclo de cosecha sea más eficiente desde el punto de vista energético.

El ciclo completo comprende cinco recorridos: entrada al lote, llenado, salida, cadeneo o transbordo y regreso al lote, el mayor consumo energético se presenta en los recorridos de salida y cadeneo porque se remolca el vagón cargado. Otro factor influyente en el consumo de energía es el sobredimensionamiento de los tractores utilizados especialmente para la operación de vagones pequeños, en las condiciones probadas en campo se utilizaron tractores de $245 \mathrm{HP}$, vagones de autovolteo o HD8000 con peso bruto $160 \mathrm{kN}$ pueden operarse con tractores de 160 a 175 HP. La Figura 7, muestra la variación del trabajo realizado por ciclo en función de la distancia de transbordo en una cosecha mecánica con tres tipos de vagones (autovolteo, HD12000 y HD20000). El trabajo se determinó con base en los requerimientos de tracción de los vagones en sus diferentes estados (vacío y lleno) y las distancias recorridas en cada ciclo completo de cosecha.
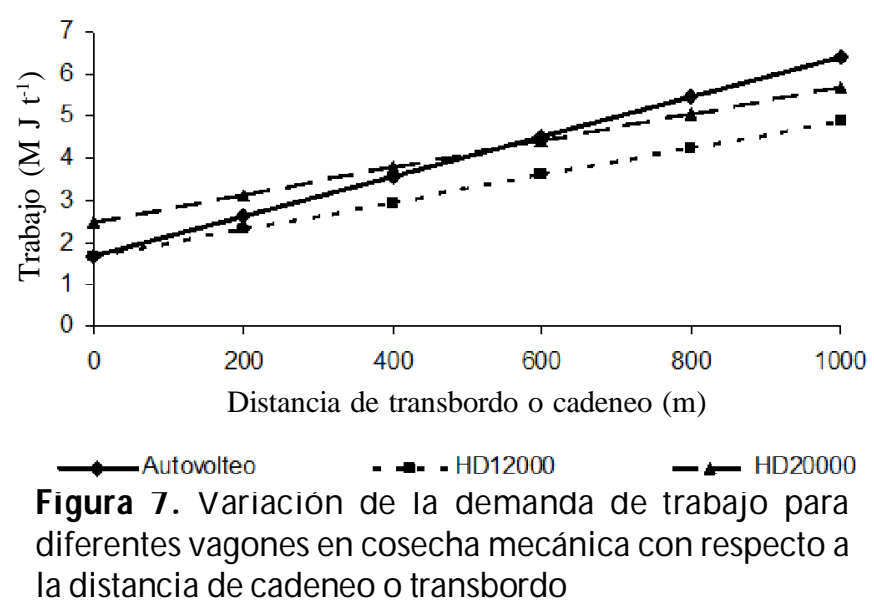

Para las condiciones de campo, el vagón HD12000 es la mejor opción con menor demanda energética, este vagón se ajustó menor al diseño de campo con recorridos muertos menores, mientras que el vagón de autovolteo es más favorable que el HD20000 hasta distancias de cadeneo o transbordo de $556 \mathrm{~m}$.

Una cosecha con manejo adecuado brinda dos condiciones recomendables de operación: recorridos muertos nulos y tractores ajustados al tamaño de cada vagón, al cumplirse estas condiciones se minimizan la IT, sus efectos y el trabajo mecánico por tonelada cosechada y aunque el vagón HD12000 sigue 
Tabla 5. Comportamiento energético de diferentes sistemas de cosecha mecánica en condiciones de campo y recomendables de cosecha

\begin{tabular}{|c|c|c|c|}
\hline Factores & Autovolteo & HD12000 & HD20000 \\
\hline Potencia utilizada en campo (HP) & 245 & 245 & 245 \\
\hline Potencia (condición recomendable) (HP) & $160-175$ & $180-200$ & $230-250$ \\
\hline Recorrido muerto campo $(\mathrm{m})$ & 245 & 141 & 210 \\
\hline Recorrido muerto (condición recomendable) (m) & 0 & 0 & 0 \\
\hline Trabajo mecánico campo (MJ t-1) & 3,09 & 2,73 & 3,56 \\
\hline Trabajo mecánico (condición recomendable) (MJ t-1 ) & 2,24 & 2,50 & 3,21 \\
\hline Consumo combustible campo $\left(\mathrm{L} \mathrm{t}^{-1}\right)$ & 0,36 & 0,32 & 0,42 \\
\hline Consumo combustible (condición recomendable) $\left(\mathrm{L} \mathrm{t}^{-1}\right)$ & 0,27 & 0,29 & 0,38 \\
\hline Emisiones CO2 campo $\left(\mathrm{g} \mathrm{t}^{-1}\right)$ & 965 & 854 & 1111 \\
\hline Emisiones CO2 (condición recomendable) $\left(\mathrm{g} \mathrm{t}^{-1}\right)$ & 700 & 742 & 1023 \\
\hline
\end{tabular}

siendo una buena opción, el vagón de autovolteo es más favorable hasta distancias de transbordo o cadeneo de $476 \mathrm{~m}$ como se muestra en la Figura 8. Esto indica que con buen manejo de la cosecha, los vagones de autovolteo con peso bruto de $160 \mathrm{kN}$, además de causar menores efectos sobre el suelo y el cultivo y mantener mayores producciones, son una buena alternativa desde el punto de vista energético y ambiental ya que el consumo y las emisiones están relacionados a los requerimientos de trabajo de los diferentes vagones o trenes de cosecha.

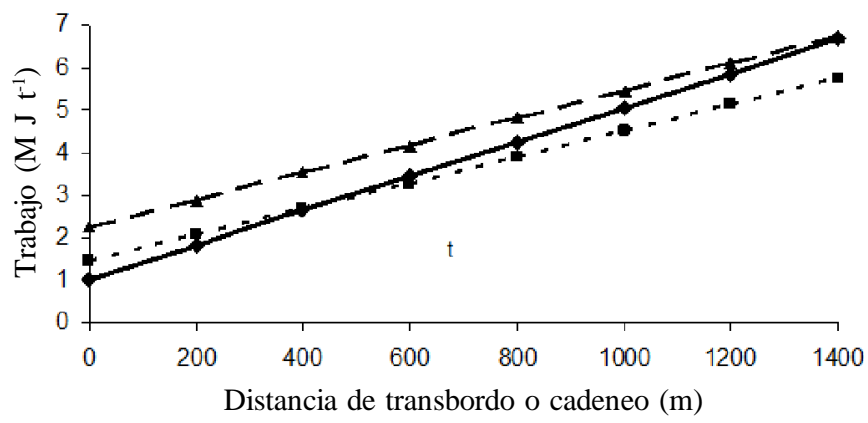

$\longrightarrow$ Autovolteo - -- - HD12000 - - HD20000

Figura 8. Variación de la demanda de trabajo mecánico con respecto a la distancia de cadeneo, para condiciones de manejo recomendado de la cosecha

El manejo adecuado de la cosecha permite reducir el consumo de combustible y las emisiones por tonelada de caña cosechada. La Tabla 5 resume los factores relacionados a demanda energética de los tres tipos de vagones con los respectivos consumos y emisiones para las condiciones de campo y recomendables de cosecha.

El consumo de combustible se determinó a partir del trabajo mecánico desarrollado por los tractores a la barra de tiro para remolcar cada vagón en cada estado del ciclo de cosecha. Dicho cálculo incluye estimativos de las pérdidas de tracción y transmisión y el rendimiento de los motores. Las emisiones de $\mathrm{CO}_{2}$ se determinan por metodologías y factores de conversión estandarizados (CIC, 2011). En condiciones de campo, el vagón HD12000 presentó menor consumo de combustible por tonelada de caña cosechada $\left(0,322 \mathrm{~L} \mathrm{t}^{-1}\right)$ y menos emisiones de $\mathrm{CO}_{2}\left(854 \mathrm{~g} \mathrm{t}^{-1}\right)$ debido al menor requerimiento de trabajo mecánico y mejores condiciones de operación respecto al lote y al tractor utilizado para operación en condiciones similares y recomendables respecto a manejo, diseño de campo y tamaño de tractores, los vagones de autovolteo son nuevamente favorables con menor consumo de combustible $\left(0,266 \mathrm{~L} \mathrm{t}^{-1}\right)$ y menor emisión de gases de efecto invernadero $\left(700 \mathrm{~g} \mathrm{CO}_{2} \mathrm{t}^{-1}\right)$.

Con respecto a los vagones HD12000 y HD20000 y en condiciones recomendables de cosecha, el vagón de autovolteo genera ahorros de combustible de 6,9 y $28,9 \%$ y reducción de emisiones de $\mathrm{CO}_{2}$ de 6,6 y $23,8 \%$ por tonelada de caña cosechada. La Figura 9 compara las emisiones de $\mathrm{CO}_{2}$ para condiciones actuales de campo y recomendables de operación.

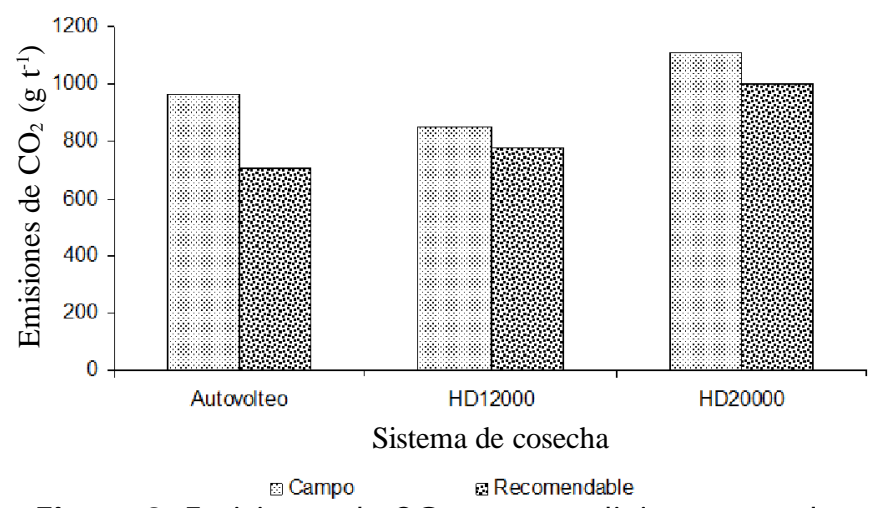

Figura 9. Emisiones de $\mathrm{CO}_{2}$ para condiciones actuales de campo y condiciones recomendables con tres sistemas de cosecha

\section{ConClusiones}

1. El efecto del tráfico de la cosecha de caña sobre el suelo y el cultivo está relacionado a la intensidad de tráfico causada por los equipos durante la labor.

2. En cosecha mecánica se presentaron diferencias en productividad hasta del 13,9\% entre el sistema con vagones pesados y de alta capacidad y el sistema con vagones livianos y de menor capacidad como el autovolteo. En cosecha semimecánica esta diferencia alcanzó hasta 4,3\%.

3. El peso de los vagones que entran al campo sobrepasa los límites de carga por eje para prevenir compactación en el subsuelo.

4. La cosecha con vagones livianos y de menor capacidad causa menor compactación tanto superficial como subsuperficial y con manejo adecuado garantiza menor consumo de combustible y menor cantidad de emisiones hacia la atmósfera. 


\section{Agradecimientos}

Cenicaña agradece al Ministerio de Agricultura y Desarrollo Rural de Colombia por su ayuda financiera y a los Ingenios Riopaila-Castilla, Manuelita e Incauca ubicados en el valle del río Cauca (Colombia) por facilitar campos y equipos para la ejecución de este proyecto.

\section{LITERATURA CITADA}

Balbuena, R. H.; Terminiello, A. M.; Claverie, J. A.; Casado, J. P.; Marlats, R. Compactación de suelo durante la cosecha forestal. Evolución de las propiedades físicas. Revista Brasileira de Engenharia Agrícola e Ambiental, v.4, p.453-459, 2000.

Botta, G.; Jorajuria, D.; Balbuena, R.; Rosatto, H. Mechanical and cropping behavoir of direct drilled soil under different traffic intensities: effect on soybean (Glycine max L.) yields. Soil \& Tillage Research, v.78, p.53-58, 2004.

Botta, G; Pozzolo, O.; Bomben, M.; Tourn, M.; Soza, E.; Rosatto, H.; Gili, A.; Ressia, J.; Rivero, D.; Vasquez, J.; Stadler, S. Aplicación del tráfico controlado en la cosecha de maíz (Zea mays L.): Efecto sobre rendimientos del cultivo y las propiedades físicas del suelo. Agro-Ciencia, v.23, p.23-29, 2007.

Braunack, M. V.; Arvidsson, J.; Hakansson, I. Effect of harvest traffic position on soil conditions and sugarcane response to environmental conditions in Queensland, Australia. Soil \& Tillage Research, v.89, p.103-121, 2006a.

Braunack, M. V.; McGarry, D. M. Traffic control and tillage strategies for harvesting and planting of sugarcane (Saccharum officinarum) in Australia. Soil \& Tillage Research, v.86, p.86-102, 2006b.

Chamen, W. C. T.; Alakukku, L.; Pires, S.; Sommer, C.; Spoor, G.; Tijink F. G. J.; Weisskopf P. Prevention strategies for field traffic-induced subsoil compaction: a review. Part 2. Equipment and field practices. Soil \& Tillage Research, v.73, p.161-174, 2003.

CIC - Comisión Interdepartamental del cambio Climático. Guía práctica para el cálculo de emisiones de gases de efecto invernadero (GEI). Generalitat de Catalunya. Oficina Catalana del canvi climàtic, Barcelona: CIC, 2011. 66p.
Hakansson, I.; Voorhees, W. B.; Riley, H. Vehicle and wheels factors influencing soil compaction and crop response in different traffic regimes. Soil \& Tillage Research, v.11, p.239$282,1988$.

Hamza M. A.; Anderson, W. K. Soil compaction in cropping systems. A review of the nature, causes and possible solutions. Soil \& Tillage Research, v.82, p.121-145, 2005.

Iaia, A. M.; Maia, J. C. S.; Kim , M. E. Uso de penetrômetro electrônico na evaluaçao da resistência do solo cultivado com cana-de-azucar. Revista Brasileira de Engenharia Agrícola e Ambiental, v.10, p.523-530, 2006.

Jorajuria, D.; Draghi, L. Sobrecompactación del suelo agrícola. Parte I. Influencia diferencial del peso y del número de pasadas. Revista Brasileira de Engenharia Agrícola e Ambiental, v.4, p.445-452, 2000.

Kroulik, M.; Kumhala, F.; Hula, J.; Honzik, I. The evaluation of agricultural machines field trafficking intensity for different soil tillage technologies. Soil \& Tillage Research., v.105, p.171-175, 2009.

Kuipers, H.; Zande, J. C. van de. Quantification of traffic systems on crop production. In Developments in Agricultural Engineering. Soil compaction in crop production. St. Joseph: ASAE, 1994, p.417-445, .

Meyer, E. The performance of machinery for mechanical harvesting and loading of sugarcane. In: Annual Congress of the South African Sugarcane Technologies Association (SASTA), 75, Proceedings... 2001. CD Rom

Nagaoka, A. K.; Lanças, K. P.; Castro, P.; Lopes, A.; Guerra, S. P. S. Resistência do solo à penetração apòs tràfego com dois tipos de pneus utilizando-se um equipamento para ensaio dinâmico. Revista Brasileira de Engenharia Agrícola e Ambiental, v.7, p.387-393, 2003.

Saffih-Hdadi, K.; Defossez, G.; Richard, Y; Cui, J. C.; Tang, A. M.; Chaplain, A. A method for predicting soil susceptibility to the compaction of surface layers as a function of water content and bulk density. Soil \& Tillage Research, v.105, p.96-103, 2009.

Terminiello, A. M.; Balbuena, R. H.; Claverie J. A.; Casado, J. P. Compactación inducida por el tráfico vehicular sobre un suelo en producción hortícola. Revista Brasileira de Engenharia Agrícola e Ambiental, v.4, p.290-293, 2000. 